carefully watched on the assumption that the case was rather one of pulmonary than of abdominal trouble. There was no definite change at 6 P.M. An enema saponis had been retained but the pain had not been relieved by poulticing. The temperature at this time was $99 \cdot 8^{\circ} \mathrm{F}$., the pulse was 120, and the respirations were 60 . At midnight there was still no material change in the condition of the patient, who $0^{\mathrm{e}}$ temperature was $99.8^{\circ}$ as before, pulse 120 , and respirations from 60 to 70 . A little liquid had been romited. At 1 A.M. the abdomen was distended and the patient was slightly collapsed with a pulse of 144 , almost running. At 2 A.M. he was still more collapsed and I saw him for the first time as I was doing the night work of one of my senior colleagues. He appeared to me to be so ill that I decided to explore his abdomen at once. I therefore opened it in the middle line above the umbilicus. Liquid contents escaped as soon as the peritoneum was incised, the stomach was drawn up into the wound, and a perforated ulcer was found apparently on the posterior aspect. The aperture was large enough to admit a full-sized probe and there was a considerable deposit of lymph round its edge. An attempt was made to close the perforation by Lembert's sutures, but the operation was very difficult, partly on account of the awkward position of the ulcer and partly owing to the rotten state of the surrounding tissues which were too softened to retain the silk sutures. Four sutures were passed and it seemed as though the opening had been closed successfully. During the insertion of the sutures there was an abundant flow of clear and thin liquid from the interior of the duodenum, but this ceased when the last sature was tied. The peritoneal cavity was then cleansed and the wound was closed. The patient bore the operation badly and died five and a half hours later. A post-mortem examination at 3 P.M. on the same day showed that the whole peritoneum was acutely inflamed, its endothelial aspect being covered with a layer of fibrino-purulent lymph. There were collections of purulent matter at the bottom of the recto.vesical pouch, in the lumbar region, and in the right subphrenic space, this space being almost completely shut off from the rest of the peritoneal cavity. A conical ulcer measuring half an inch at its widest part was situated at the upper and back part of the first portion of the duodenum. It had sharply-cut edges but there was no infiltration of its margins. The floor of the ulcer had perforated but my sutures were not applied accurately because water and intestinal contents passed easily through the perforation.

The particular interest of this case lay in the fact that the localised peritonitis must have lasted a much longer time than the sudden onset of symptoms would have led one to suspect, whilst the symptoms when they appeared were so obscure as to make it appear to competent observers that the patient was suffering from pneumonia rather than from peritonitis. It is interesting, too, because it is a record of the course taken by a case of perforated duodenal ulcer which, though carefully watched, was practically untreated and it shows the suddenness with which a patient in this condition passed from comparative ease into the jaws of death.

The prognosis in cases of duodenal perforation is bad and the average mortality is much higher than in perforation of the stomach. Many die from shock, some from peritonitis, others from pneumonia ten days or a fortnight after the perforation and just as the surgeon is beginning to hope that he has got his patient beyond any fear of a relapse. The prognosis of duodenal perforation will improve as the condition is more generally recognised and its special dangers are more widely understood. Perforated gastric ulcer had as bad a prognosis a few years ago, though the signs which accompany it are far plainer than those of duodenal perforation. Gradually we have become alive to the facts and have learnt that immediate operation is followed by recovery in most cases, whilst to wait almost certainly means death to the patient. Cases are now sent to us much more quickly than they used to be-indeed, the last man I operated upon was at work at half-past twelve one morning, had signs of gastric perforation at one o'clock, and by three the same afternoon I had operated upon him and he was safely back in bed. It is needless to say that he recovered without a bad symptom. The advice that $I$ would offer you, therefore, in cases of duodenal perforation is to operate at once when you have reasonable grounds to suppose that such a catastrophe has taken place. Do not wait until you have definite signs to depend upon. The pulse, the temperature, and the degree of movement of the abdominal walls may all deceive you. The patient will recover from the initial shock if he be watched and yet all the time his peritoneum is becoming more and more involved, so that valuable time is being lost if you allow his general condition to weigh against the advisability of an operation. When you are in doubt examine the abdomen. Fulness and tenderness over the appendix region should not mislead you, but you should at once ascertain whether there is not an equally tender spot with resistance higher up and on the same side of the abdomen over the situation of the duodenum. If there is such a tender spot and it be associated with the other signs and symptoms of duodenal perforation then operate without more ado, for in this way, and in this way only, you will reduce the mortality to a minimum.

The operation will be difficult or easy according to the position and surroundings of the ulcer which has led to the perforation. A duodenal ulcer is usually conical, its apex is directed outwards, and the actual perforation therefore is small, often no larger than the point of a probe. One or two sutures may be enough to close it. It is situated in the majority of cases in the first part of the duodenum, close to the pylorus and on the upper and anterior wall. The more difficult cases, some of which it is impossible to close by suture, are those situated on the posterior aspect of the duodenum or in its second or third parts as well as those where the intestinal wall is softened by inflammatory products or is surrounded by adhesions. The perforation is closed with sutures of No. 1 silk inserted with a quartercurved needle after the intestine has been brought as close to the surface as possible; when it is impossible to bring it into the wound I have often found a cleft palate needle and cleft palate forceps serviceable, so I have these in struments put out for me whenever I have a case of gastric or duodenal perforation to operate upon. When the perforation is closed and the intestine has been replaced in the abdominal cavity a large drainage tube without any holes in the sides is passed into the abdominal carity and the incision is closed in the usual manner. I then open the peritoneal cavity in each iliac region as a matter of routine and insert a large drainage-tube on each side There is nearly always a considerable escape of fluid from these lateral incisions and they continue to discharge longer than the first wound over the duodenum. The tabes are emptied twice a day by sucking up their contents with a male glass urethral syringe to which a small rubber tube is attached. It is a better plan than that of taking the tubes out and syringing the sinus. The tubes should be dispensed with as soon as possible, because adhesions quickly form round them and shut them off from the general cavity of the peritoneum and they then serve no good purpose but act as irritants and increase the amount of discharge. They may be removed within the first week and the wounds are then packed lightly with gauze. When the patients recover they do so perfectly, for they do not seem to be subject to the relapses which are so common in cases of perforated gastric ulcer. One other point and I have finished. Duodenal perforation occurs four times more often in men than in women, and no one knows why.

\section{THE SACCULAR THEORY OF HERNIA AND THE RADICAL OPERATION.}

BY R. HAMILTON RUSSELL, F.R.C.S. ENG.,

SURGEON TO THE ALFRED HOSPITAL, MELBOURNE; CONSULTING SURGEON TO THE MELBOURNE HOSPITAL FOR SICK CHILDREN.

By the saccular theory of hernia I mean the theory that rejects the view that hernia can ever be "acquired" in the pathological sense and maintains that the presence of a developmental peritoneal diverticulum or sac is a necessary antecedent condition in every case of ordinary abdominal hernia. In the numerous articles which I have contributed to THE LANCET during the last seven years on this subject ${ }^{1} I$ have always been content to speak of the "congenital origin" of hernia or of "hernia into a congenital sac"; but for reasons that I have pointed out on several previous occasions there are special inconveniences associated with the use of the word "congenital" in connexion with hernia, and I am therefore prompted to suggest a name which has

1 The Lancet, Nov, 18th, 1899, p. 353; Oct. 20th, 1900, p. 1128 ; May 31st, 1902, p. 1519; March 12th, 1904, p. 707; and Jan.7th, 1905, 
the great advantage of being at once unobjectionable and appropriate.

I think I am entitled to say that for the moment there exist two schools of surgical opinion in respect of hernia. There is a small school of surgeons who are convinced of the truth of the saccular theory of hernia, and a very large body of surgeons who do not as yet see sufficient reason for so radical a change of opinion. Of these again I am sure I am safe in saying that a large number are experiencing some feeling of interested doubt; to give a stimulus to that feeling and perchance contribute something towards the resolution of that doubt are among the main objects of this article. I regard it as impossible that so wide a division of opinion as this can be other than a very transient episode; it is certain that the saccular theory will be either universally accepted or universally rejected in the near future.

In all the more recent communications that $I$ have made to THE LANCET I have laboured to prove the truth of the saccular theory by directing attention to the significance of a great body of clinical facts and the deductions from them that are forced upon us in response to accurate observation and careful reasoning. I have pointed out that there is not a single fact at our disposal which, if scrutinised closely enough is not at once in revolt against the traditional view as to the etiology of hernia and in complete harmony with the saccular theory. On the present occasion I do not intend to pursue the subject further on the same lines as heretofore. The saccular theory will fail or prevail as it is false or true ; it will not, however, perish from want of recognition. This is rendered assured by the admirable contributions which have been from time to time made to the subject $b_{j}^{m}$ Stiles, Deanesley, R. W. Murray, and other surgeons; and notably by the result of the series of necropsies performed at the Mill-road Infirmary, Liverpool, at Murray's instigation, and communicated by him in a paper to THE LANCET. ${ }^{2}$ In this investigation 100 bodies were examined, 61 males and 39 females. Of the 61 males 13 bodies showed potential hernial sacs, the total number of sacs being nine inguinal and 12 femoral ; two sacs contained omentum. Of the 39 female bodies hernial sacs were found in eight, the total number of sacs being nine femoral and one umbilical; two of these sacs contained omentum. This preponderance of femoral sacs is a surprise to me and opens up questions of interest that call for further inquiry ; I will not stay to allude to them here.

With reference to these pathological observations I cannot forbear to call attention to a sentence that will be found in a paper which I published in THE LANCET ${ }^{3}$ in 1902: "I would suggest that if some of our pathological friends who have the opportunity of making large numbers of post-mortem examinations would examine the hernial orifices in a large number of bodies they would be enabled to make some very valuable and interesting observations with reference to this matter." This, which sounded at the time a most unwarrantable and reckless statement, was not so in reality. Apart from the fact that the clinical evidence alone would be inexplicable I had seen such sacs in bodies. Moreover, I was immediately furnished with very interesting evidence that such sacs must have been of ten seen by other observers, but with eyes that seeing yet saw not; and this, not from want of skill in accurate observation, but from inability to attach any significance to the incident because no significance chanced to occur to them and none had ever been suggested. The sentence quoted was uttered at the Hobart meeting of the Australasian Congress in 1902. Out of my audience of perhaps 20 to 30 medical men two surgeons were able to recall the fact of having observed such sacs. One distinguished observer, Professor A. Watson of Adelaide, had observed a femoral and an obturator sac co-existing; another surgeon remembered to have once by chance seen a femoral sac. Neither of these gentlemen had been able to attach any special significance to the fact, which had lain slumbering in the storehouse of the memory until suddenly aroused by my suggestion as to the essential part played by such sacs in the production of hernia.

It has occasionally been intimated to me that the practical purport of the saccular theory is not very evident. I have even been asked, "What does it matter?" I purpose in this paper to answer that question, and as a preliminary I wish to point out the defects in our present position with regard to hernia. They are glaring and obvious, but we have become habituated to regarding them with equanimity. 'They are as follows : (1) A double etiology (congenital and acquired) with resulting uncertainty as to the fundamental principle of treatment; (2) a multiplicity of operations, an infallible sign of vagueness and uncertainty of principle; (3) uncertain results of operation due to uncertainty as to the principles governing it; (4) a fatal facility for explaining away failure as being due to inherent weakness of the muscular structures in the inguinal region (this explanation is always fair and quite warranted by the accepted but erroneous views as to the etiology of hernia); and (5) the misuse of the truss, notably in the case of children. Here at any rate is a field that offers scope for improvement, and I shall now state what the saccular theory will do for the surgery of hernia, following the above five clauses seriatim. It will have for results : (1) a single etiology, with a fixed principle of treatment; (2) a single operation, the removal of the sac ; (3) certainty of good result provided the operation has been performed faultiessly ; (4) the recognition that if recurrence takes place it will be the result of an operative defect ; and (5) the abolition of the truss except for cases in which operation is contra-indicated or declined. It is not pretended that this is a modest undertaking, and in order to support it $I$ shall be so venturesome as to rewrite largely the main essentials of the theory and practice of surgery in respect of hernia, showing in what way and to what extent our views, first, as to the etiology and classification of hernia, and finally, as to the operation for its radical cure, will in my opinion be altered by the saccular theory. Let me hasten to explain that there is nothing visionary or prophetic in what I am about to write. There is not, I believe, a word in the section of etiology and classification that is not in substance inevitable; there is certainly not a word on the subject of the radical cure that is not based on the record of my own practice and personal observation. Let me finally add that in this paper the truth of the saccular theory is assumed, and that where any statement is made unsup. ported by any reasoning and in violent conflict with accepted views the arguments in support of it will have been fully dealt with in previous papers.

General Classification of Abdominal Hernid.

There are three groups of abdominal hernia which I will distinguish in their order of frequency as $(a)$ saccular, $(b)$ traumatic, and (c) congenital. Of these three groups the saccular form comprises all cases of ordinary hernia, and will therefore include all the varieties of inguinal, femoral, and umbilical hernia, together with the less commonly seen varieties, the obturator, ischiatic, \&c. Traumatic hernia will indicate the hernial protrusions seen after injuries of the abdominal wall, notably after surgical operations. Con. genital hernia, a term that has been the source of endless obscurity and confusion in the past, will find its proper sphere in indicating only such forms of hernia as are actually present at birtb. Such are those that result from congenital imperfections in the abdominal wall in the middle line; or elsewhere, mostly in the lumbar region as the result of congenital insufficiency of the musculatare. The term is also applicable to certain cases of saccular hernia in which the hernia is present at birth. No confusion will be caused by using the adjective "congenital" in this way; and the great inconvenience to which we have been subjected in the past by the use of the term to signify a form of inguinal hernia that frequently makes its first appearance late in life, and that is no more congenital that any other form of saccular hernia, will pass from us. For the present I propose to deal only with the saccular varieties of hernia and their treatment by operation.

\section{SaCcular Hersia.}

Saccular hernia is a term that covers all those forms of abdominal hernia which result from the descent of some portion of the abdominal viscera into a sac. The sac upon which the occurrence of hernia will be ultimately dependent is a peritoneal diverticulum of developmental origin, and its formation is to be traced back to an early period of embryonic life. It is seen with especial frequency in situations of close proximity to important vessels that pass outwards from the abdomen into the limbs, such as the femoral, the obturator, and the ischiatic vessels ; its presence is doubtless due to the accidental involvement of a portion of the wall of the primitive pleuro-peritoneal cavity in the developing limb. In the inguinal canal the hernial sac is provided by a peritoneal diverticulum that is normal and purposeful, the processus vaginalis testis. This under the 
most favourable conditions should be obliterated soon after the descent of the testis. Failure of obliteration, partial or total, together with infinite variety and irregularity in anatomical arrangement, provides the surgeon with more raried and interesting material in the inguinal region than in any other of the seats of hernia; and it will be with the rarieties of inguinal hernia that I shall mainly deal.

The etiology of inguinal hernia.-There are two etiological divisions of inguinal hernia, the funicular and the nonfunicular. Of these the funicular process provides the enormous majority of inguinal herniæ, while only a very small minority are non-funicular. Inguinal hernia is also divided into the classes of oblique or external and direct or internal. Of these the enormous majority are oblique, while direct hernia is relatively infrequent. The etiological relationship of these two classes is as follows. The whole of the large group of oblique inguinal hernize are of funicular origin and in addition a proportion of the direct group is also provided by the funicular process. Of non-funicular origin there will thus be only a moiety of the small class of direct herniæ. This anatomical and etiological relationship may be conveniently expressed diagrammatically thus :-

nguinal Hernia

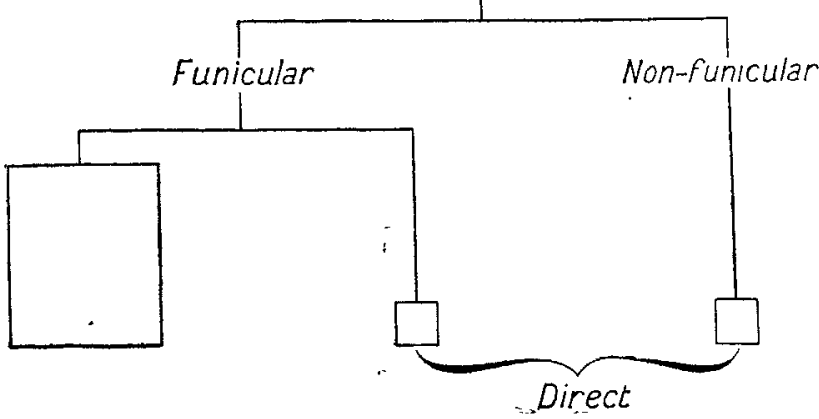

Some idea of the relative frequency of occurrence of the oblique and direct varieties of inguinal hernia may be conreved by the statement that in an experience of nearly 300 cases of hernia in children and adults $I$ have met with direct hernia only three times; on two of these occasions the hernial sac was of non-funicular origin. Thus by sheer force of numbers and of infinite variety the anatomical class of oblique inguinal hernia takes a predominant positicn as compared with the direct; while even more comprehensive is the etiological class that owns a funicular origin, for this will include not merely the whole of the oblique inguinal hernix, but in addition the most interesting and important section of the direct.

The processus vaginalis and the varieties of funicular hemia.-Where the formation of the processus vaginalis and the descent of the testis have proceeded normally, the serous covering of the testis will be continuons with the peritoneum by a slender tube of serous membrane passing through the length of the inguinal canal. Obliteration of this tube should now take place from the point where it debouches into the peritoneum above, to a spot vear the testis below. It is at the upper end of the funicular process that the progress and accomplishment of obliteration assume features of special interest to the surgeon and of importance to the future of the individual. When the peritoneal tube is obliterated fairly up to the level of the opening in the transversalis fascia the occurrence of inguinal hernia in the future will be impossible. Failure of obliteration in the upper portion of the tube will leave a peritoneal diverticulum the magnitude of which will depend on the extent to which the obliterative process has fallen short. All varieties of oblique inguinal hernia owe their occurrence primarily to failure of obliteration in the upper portion of the funicular process, while their various anatomical characteristics are all of them determined by accidental variations in the anatomical arrangement of the funicular prosess itself. There are thus presented by the funicular process two distinct groups of phenomena for examination. Of these one group is dependent upon variability in the process of obliteration, while the other is governed by primary anatomical variations of developmental origin. To these two groups must be added a third, which I have called "viscero-saccular hernix"; this group will comprise an important class in which the formation of the funicular process has been attended by the dragging of some neighbouring viscus or portion of a viscus into the inguinal canal. These three groups will now be examined in some detail.
Group 1. Tariations due to obliterative failure.-These are exceedingly simple. In Fig. 1, A represents the funicular process, open throughout, as it is normally on the completion of the descent of the testis. In $B$ there has been failure of obliteration in the upper portion of the funicular process, leaving a diverticulum from the peritoneum. c, obliteration perfect, rendering the occurrence of hernia impossible. There are two varieties of inguinal hernia that owe their

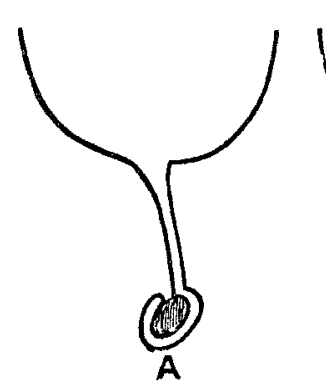

The dotted line represents the obliterated portion of the funicular origin solely to obliterative variations, and they are indicated in $A$ and 3 . (a) Complete failure of obliteration, so that the funicular process remains open throughout (Fig. 1, A). The resulting hernia will be an example of total funicular hernia. This has been called indifferently "congenital hernia" or "hernia into the tunica vaginalis." The former term must be abandoned as applied to this variety of inguinal hernia, and the latter does not seem to be well chosen. (b) Failure of obliteration in the upper portion of the funicular process (Fig. 1, B). The resulting hernia will be a partial funicular hernia; this has hitherto been known as funicular hernia. These two varieties, cotal and partial funicular, are the only varieties of hernia proper that depend upon mere obliterative variations in the funicular process. The number of possible variations of this character are infinite, but they are capable of producing only variations in the size of the partial funicular hernia on the one hand, or, on the other, of bringing about various clinical types of hydrocele, either hydrocele of the tunica vaginalis or of the cord, which may or may not be associated in addition with a partial funicular hernia.

GRoUP 2. Primary anatomical rariations due to derelopmental acoidents. - 'The embryonic period at which the fanicular process is formed is one of extreme activity and rapid change, and the slender tube of peritoneum is at this time imbedded in a mass of mesoblastic tissue that is performing rapid evolutions in its progress towards the ultimate formation of a perfect inguinal region and scrotum. As a result of this circumstance apparently we find that the funicular process becomes, as it were, caught here and there by the neighbouring structures and dragged by them into anomalous and unusual positions. Lockwood has directed attention to the part probably played in this process by a quantity of involuntary muscular tissue which is distributed over this region of the peritoneum, and of which certain portions are distinguishable as the gubernaculum -testis. This is doubtless the correct explanation of the great variety of secondary sacculations and other irregularities that are observed in the funicular process with extreme frequency; nor are these phenomena peculiar to the funicular process, for they are observed with fully equal frequency in the case of the femoral sac. To this proneness to irregular sacculation of the funicular process we are indebted for all the named varieties of inguinal hernia other than those I have already alluded to as total and partial funicular. I will briefly indicate the important positions such secondary sacs are liable to adopt. In the following descriptions it will be understood that the sac, in every instance, is a single diverticulum from the wall of the funicular process, and that the main tube of the funicular process may be either completely open, or completely closed, or partially closed. The infinite variety of the possible clinical combinations will be obvious. Fig. 2 a represents what I believe to be the most frequent form of secondary sac. It will be seen springing from the inner side of the hernial sac high up but below the epigastric artery. The hernia will usually enter the main portion of the funicular process ; I have known, however, a hernia to come suddenly down into such a secondary sac, while the funicular process was open throughout as $I$ have here represented it. Fig. $2 \mathrm{~B}$ represents a form of bernial sac closely akin to the last but of extreme interest and 
surgical importance. The sac comes off from the funicular process high up and descends on the inner side of the epigastric artery. Hernia descending into this sac will therefore be a direct or internal hernia of funicular origin which we must name direct or internal funicular hernia. It is fortunately somewhat rare and the treatment is difficult and will receive

FIG. 2.

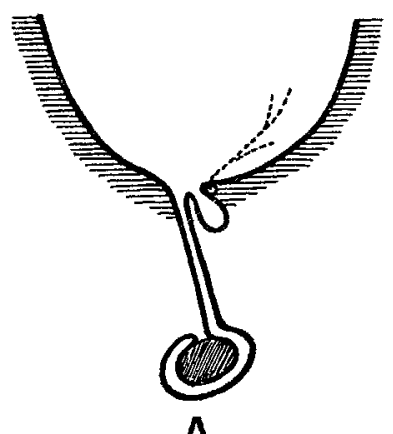

A
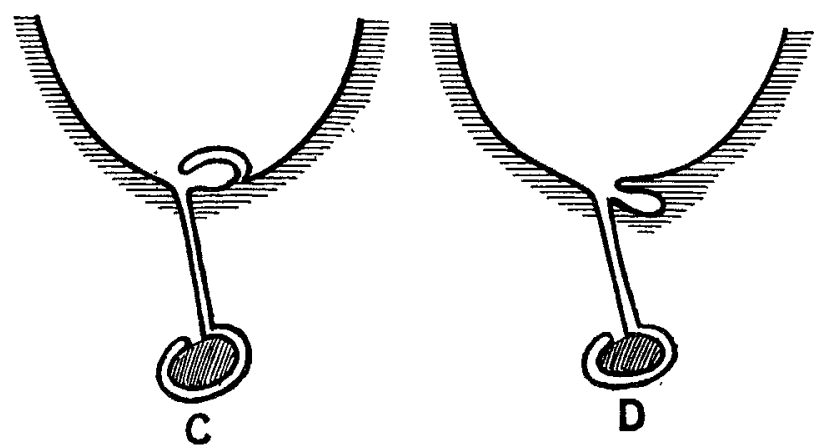

In $A$ and $B$ the position of the deep epigastric artery is indicated by a clotted line.

special notice later. The close kinship between these two varieties and the sacs of interstitial and properitoneal hernia (C and D) will be noted. To these it is hardly necessary to add a diagram showing a position of the sac frequently seen-viz., on the surface of the aponeurosis of the external oblique. These three last forms of hernia are often seen in conjunction with imperfect descent of the testis ; this has seemed to me to apply more especially to the last-named of the three. It has been thought that the imperfectly descended testis might, by acting as an obstruction in the canal, be causative in the production of interstitial and properitoneal hernia; this view is at once disposed of by the consideration that, in the first place, interstitial and properitoneal hernia may occur without any association with retained testis, and secondly, that the sac of interstitial or properitoneal hernia is commonly found in cases of imperfectly descended testis in which no hernia has ever been present.

The foregoing description of various kinds of anatomical variation has dealt with cases that are clinically unusual by the fact that the sac occupied by the hernia is an offshoot from the normal one, the funicular process proper. There is yet another group of anatomical irregularities in which there are irregular dispositions of other portions of the funicular process which obscure the normal relationships of the hernial sac and are apt to cause embarrassment to the operator. I observe that it is still the custom of authors to discourse upon these varieties under the heading of "infantile" hernia, a term that I think might with advantage be suffered to lapse. Much light has been thrown upon the mode of origin of these varieties by Lockwood. The subject is too extensive to be conveniently dealt with here, and I have nothing to contribute in furtherance of the immediate objects of this paper.

Group 3. Developmental accidents resulting in the implication of abdominal organs in the formation of the funicular process. - The mode of origin of this group of hernial variations is readily understood if we cast back to the embryonic period at which the sac is formed. In the course of the events that result in the inclusion of a portion of the peritoneum in the inguinal canal and scrotum, it not infrequently happens that one of the abdominal viscera which receive a peritoneal covering will be encroached upon. In this way a portion of the bladder may be drawn into the canal with the sac. On the left side the terminal po:tion of the descending colon and the commencement of the sigmoid are sometimes seen, while on the right side (in rare cases, on the left) the cæcum may appear under these conditions. It is clear that the protruded viscus in these cases will have a special relation to the hernial sac, which relation will be quite distinct from that of a structure berniated in the ordinary way. Thus, in a hernia of the bladder the bladder will not be in the sac but of the sac; actually outside of it but virtually contributing to the formation of it. The same will apply to this particular description of hernia of the cæcum and the sigmoid. The relationship between the herniated organ and the sac in these cases will be allnded to again in the description of their operative management. It is only needful here to remark that the relationship of these various structures to the sac is not necessarily that which $I$ have here described. It is possible for the sigmoid flexure or the cæcum to be quite free and unattached in a hernial sac. It is also stated by some anthors that even a portion of the bladder may be actually inside the sac. I have never myself observed anything like this in the case of the bladder and $I$ find difficulty in conceiving its occurrence.

Congenital hernia of the omentum.-This is a variety of hernia sui generis, and I wish to allude to it here on account of its importance which does not seem to me to have been quite appreciated. It is seen in its most interesting form in young children when it often causes difficulty in diagnosis. It also frequently fails to attract the notice of the child's parents, hence it is seen in operations later on in life. It is usually present as a long, thin strip of omentum passing down the funicular process, to which it will sometimes have contracted an adhesion. I have observed instances of both total and partial funicular sacs occupied by a strip of omentum in this way. There is no doubt that its presence in the funicular process is the result of a developmental accident, and the affection is, in the proper sense of the term, congenital. I have formed a very strong opinion that in the large majority, if not in all, of the cases in which omentum is found during operation at any period of life as one of the constituents of a hernia, the omentum has been present at birth. The chain of events is obvious, for when an individual has harboured an omentum. containing sac for years without being aware of it, the occurrence of an enterocele will not convey to him or to the surgeon any suggestion of the antecedent condition. On the other band, in operating upon adults, I have so often met with the curious long strip of omentum with which I have become familiar in the congenital omental herniæ of children that I could have no doubt as to its congenital origin. I am further impressed on reflection by what would appear to be extreme improbability in the belief that a piece of omentum should be the first structure to insinuate itself into a hernial sac; or again that it should ever succeed in worming itself past the intestine into an enterocele already formed. It is noteworthy in this connexion that in Murray's series of postmortem examinations above alluded to a piece of omentum was found in the potential hernial sac on four occasions.

Femoral hernia.-I mention this form of hernia in order to point out, not for the first time, a most interestirg result of the saccular theory in explaining the frequent ascent of femoral hernia over Poupart's ligament. No explanation worthy of the name has ever been offered of this remarkable phenomenon; but the reason for its occurrence becomes at once evident when we learn that the sac of a femoral hernia is a congenital structure. I have explained this very fully in THE LANCET on two previous occasions. ${ }^{4}$

The radical cure of hernia.-I do not propose to give here a formal description of the anatomy of the inguinal canal. I would, however, venture to call attention to what I conceive to be the salient features of its protective mechanism against the occurrence of hernia. Let us view it as composed of two layers; a substantial muscular layer externally, lined by a slender peritoneo-fascial layer internally. The structures of the cord curving upwards above Poupart's ligament pass first through the transversalis fascia, by which they are closely embraced, and which is carried down on the cord forming its most intimate covering. The opening in the fascia that permits the exit of the cord from the abdo. minal cavity is the internal abdominal ring. From its entrance into the canal at the internal abdominal ring the cord passes obliquely through the abdominal wall, closely embraced by the muscular layer, until it emerges at the 
external abdominal ring. In considering the respective parts played by these two layers of the abdominal wall in the prevention of hernia it is obvious that the entire burden must fall upon the muscular layer. It will be seen that while the fibres of the internal oblique and transversalis spring from the outer two-thirds of Poupart's ligament, they pass to be attached through the medium of the conjoined tendon to the bone of the pubic ramus at a lower level than Poupart's ligament. 'Their contraction, therefore, will result in an effect on the canal in the nature of that of a sphincter. Or if it be objected that the nature of this muscular action hardly warrants the use of the term sphincter, " a thing that closes," it will at least be conceded that it is entitled to that or any other term appropriate to a thing that "prevents opening." Hence it is convenient for want of a better word to speak of it as a sphincter. The production of hernia is dependent on such increase of the intraabdominal pressure that the retentive power of the abdominal wall fails at some specially weak point. Such increase of internal pressure is, with very rare exceptions, dependent on some muscular effort performed by the individual; in the absence of such effort the intra-abdominal pressure may be regarded as practically nil. Hence it is during the performance of some arduous feat or the lifting of a heavy weight that hernia is most prone to occur. Now the anatomical arrangement of the inguinal sphincter provides that any effort such as to endanger the inguinal canal will be resisted with a force in direct ratio to the effort expended; it therefore constitutes an automatically acting protective mechanism exactly adjusted to the requirements of the moment Were there no other protecting mecbanism than this watching over the inguinal canal it is extremely improbable that inguinal hernia could ever occur; but there is an abundance of additional security. The whole region is powerfully supported by the external oblique aponeurosis except at the external ring, which, on the other hand, is effectively shielded by the conjoined tendon and the rectus; while the internal abdominal ring is supported in addition by the lowermost fibres of the internal oblique as they spring from their attachment to Poupart's ligament. Thus a normal inguinal canal is fortified against the occurrence of hernia to a perfection and a degree of strength in extravagant excess of any demand that can be made upon it by the feeble intra-abolominal pressure. That this is not a mere matter of theorising is proved by the fact that even the presence of a sac in the form of an open funicular process is not necessarily productive of hernia. When, bowever, we turn from the muscular layer of the abdominal wall to the peritoneo-fascial layer we find there the key to the situation. The presence of a peritoneal diverticulum into the canal is alone able to place the muscular apparatus at so great a disadvantage as to render the occurrence of hernia possible. Upon the foregoing considerations are based the two following propositions which are fundamentals in the operative treatment of hernia. (1) Where there is no developmental sac such as is provided in the case of the inguinal canal by incomplete obliteration of the funicular process, there can be no hernia; (2) the normally formed inguinal canal is endowed with a strength and retentive efficiency for resisting hernia, enormously in excess of any demand that can be made upon it by the mere intra-abdominal pressure unaided by the presence of a sac.

Of these two propositions the second would perhaps be more correctly designated an explanation rather than a guiding principle, for it finds its main field of usefulness in rendering intelligible the success of removal of the sac even after the icguinal region has been extensively damaged by the prolonged existence of hernia. Hence it will follow as a logical conclusion that early removal of the sac after the appearance of a hernia will constitute a radical cure of the affection, and that recurrence will be impossible. It will also follow from the second proposition that late removal of the sac after the abdominal wall has sustained much injury will probably prove a radical cure, and that recurrence will be unlikely to take place. I am of opinion that this last statement errs in the direction of caution; for my belief is that recurrence in even the most unfavourable class of old-standing hernia will be so rare as to be practically unknown after removal of the sac has been performed with all due care and in the best possible way. At the risk of appearing to begin my description at the wrong end I will illustrate this by the case of a man, aged 75 years, who suffered for 30 years from double inguinal

hernia, small and reducible on the left side, very large and irreducible on the right. I operated on the right side at his special request in October, 1904, removing an enormous sac and a large quantity of omentum. He wore a single truss on the left side atter the operation. He was last seen a year after the operation when the result was apparently perfect. The operation consisted in removal of the sac alone with the precautions indicated in the following description. Fig. 3 is designed to show the large hole in the abdominal wall into which the canal becomes converted in such large old-standing herniæ as this. Surgeons will recall the appearance after the sac has been tied off in the ordinary way. There is a considerable area of the peritone 0 fascial layer exposed, and it is customary to cover this over by drawing the muscle down over it by means of sutures to Poupart's ligament. This makes a nice-looking operation, but let us for a moment criticise it. In the tirst place I would point out that, on the face of it, the sac has not been completely removed. The boundaries of the mouth of the sac lie under cover of the arched fibres and Poupart's ligament; hence the whole of the area of peritoneum and fascia exposed in the aperture belongs to the sac and should have been removed with it. Had this been done it is obvious that the hole in the abdominal wall would have been closed, or nearly closed, thereby. My own method of doing this is by means of a form of ligature which is fixed at the four points indicated in the diagram by the letters $A, B, C, D$. It is inserted into the peritoneo-fascial layer under cover of the muscles, and when tightened will draw the entire periphery of the exposed area

FIG. 3.

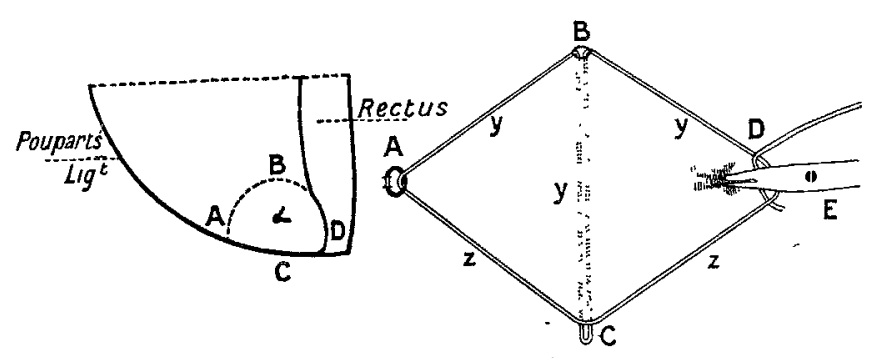

In Fig. 3 A, B, C, D indicate the points of fixation of the submuscular ligature.

In Fig. 4 A, B, C, D correspond to the points thus indicated in Fig.

into the knot. This having been done it will probably appear to the surgeon unnecessary to suture the muscular aperture, although, doubtless, no harm would be done by one or two catgut sutures lightly introduced; they would be, bowever, quite unnecessary and useless, and therefore better omitted. If now we contrast this procedure with one of the customary suturing methods, it will be seen at once where the difference lies. To start witb, in the latter case the sac will have been incompletely removed and the hernial aperture closed by closure of the outer muscular stratum of the abdominal wall. By the method I have described the sac will have been completely removed and the hernial opening closed by closure of the deep or peritoneo-fascial layer. There can, I think, be no case for discussion as to which of the two methods is the more correct in principle. The knot I have mentioned is shown in Fig. 3 . It is easy to apply and has the advantage that it completely incloses the area, while fixed at the points $A, B, C$, and D. The ligature is mounted first on an ordinary curved needle and is passed through the peritoneo-fascial layer at $A$ well out under the muscles and tied once, leaving the two ends $Y$ and $z$ long. By means of a carrier a loop from $Y$ is passed through from $B$ to $C$, leaving a long end hanging from $B$. The end $Z$ is now passed through the loop at $C$ and the ends $Y$ and $Z$ are finally tied behind a pair of pressure forceps applied at the point $\mathrm{D}$. When the ligature is tightened the four points $A, B, C, D$ will converge to the knot. It is a simple matter to test the efficiency of this knot on a piece of cloth.

I have described this methcd of operating at what may appear to be undue length, because the principle which it illustrates is the fundamental principle that governs the successful treatment of hernia by operation. This principle may be briefly expressed as closure of the hernial opening by means of the peritoneo-fascial layer of the abdominal wall and $\mathbf{n}$ st by the muscular layer, which will take care of itself. The success or failure of any mode of operating will depend directly upon the extent to which the surgeon is guided by this principle and the acuteness with which he realises that 
recurrence, if it takes place, will be due to the peritoneofascial layer alone and that all his attention must ba directed to that. As a matter of fact, it is only in such old-standing cases as that we have just considered that the fascia is included in the ligature. In more recent and favourable cases of hernia the fascia is pushed aside and the ligature is applied to the peritoneum alone. This will be illustrated by the following brief description of an oparation for recent simple hernia.

Operation for recent partial funicular hernia.-The sac having been isolated is firmly pulled upon by the left hand aided by a pair of pressure forceps, while the right hand strips the fascia upwards from its neck with a dissecting forceps. By thus freeing the sac from the fascia the peritoneum can be forcibly pulled down to the utmost limit. If the neck of the sac be now obviously clear from any complicating structure it is ligatured as high up as possible and dropped back. The ligature I employ in a simple case is usually a simplified form of the knot I have just describedi.e., simplified by the omission of the initial tie at $A$ and of the pressure forceps at $D$. It will be noted at once that in this proceeding, by clearing the fascia from the neck of the sac, the peritoneum can be reached at a higher level; and after the sac has been cut away and the stump allowed to drop back it will lie well witbin the layer of fascia which will close over it, and a mere scar in the fascia will be left.

The only variation in technique that I need allude to is that appropriate in cases that are not very recent, and in which there is a tendency for the peritoneum of the inguinal fossæ to sag out of the wound while the sac is being pulled down. Where this occurs I remove the sagging peritoneum along with the sac by means of the following manouvre. The sac is opened and the left index is passed into the abdominal cavity and into the inguinal fossæ, and the structures now lying upon it are lifted firmly upwards out of the wound. The epigastric vessels are exposed by stripping the fascia and fat inwards with a forceps, and the vessels themselves are carefully pushed inwards over the finger-tip until all the loose peritoneum of the inguinal fossæ is brought external to the epigastric vessels and appears on the finger as a kind of secondary sac. This is now included with the hernial sac in the ligature and removed. There is one other complication frequently encountered during the performance of this operation. The bladder will often appear during the process of clearing the peritoneum on the finger. It is important to be on the watch for the muscular coats of that organ and should it be observed it can be readily peeled back for a short distance. Should it not be recognised there will be danger of its inclusion in the ligature. I have heard of such an occurrence as a urinary fistula after operation for hernia. I desire here to add a word of caution which I beg may be carefully noted. The mode of operating I have just described is applicable to adults alone ; in no circumstances must any such procedure be attempted in the case of a child.

Operation in total furicular hernia.--Where the funicular process is patent throughout the method of procedure in isolating the sac will be somewhat different from that described above, because the sac will not at first be seen. Where this is the case the right course to pursue is to find and isolate first the vas and then the vessels of the cord, and then take the remainder which must contain the funicular process on the palmar surface of the left index finger while the vas and vessels hang down on the dorsal aspect. Cut across the portion on the palmar surface and see the open mouths of the divided funicular process, sejze the upper one with pressure forceps, and proceed to strip it upwards and remove it within the internal ring as before described. The lower portion of the funicular process which is continuous with the tunica vaginalis may be disregarded.

Operation in direct funicular hernia.-The existence of this form of inguinal hernia has, I believe, been overlooked, and consequently the management of it does not appear to have been studied. I have no doubt that many of the cases of inguinal hernia that have defied repeated attempts at cure on the part of skilled operatcrs are of this nature. The surgeon will probably first suspect that he has to deal with such a case when, having exposed what appears to be an ordinary inguinal sac, he finds himself unaccountably unable to isolate and free the neck for the application of the ligature. He will then notice that it is not in the usual close relationship with the cord, and on introducing a finger into the sac he will detect the epigastric vessels on the outer side. I have met with but one case of this kind in which I regret to say that I consciously failed because I was unprepared with any plan for meeting the diffculties that were presented and which were new to me. I am quite confident however, that they might have been easily overcome. It will be obviously right for me to defer any description of the method of procedure I should propose until it has been subjected to the test of experience. Meanwhile, I may be permitted to point out broadly the causes of failure and the principle that must be followed in order to ensure success. A glance at the diagram (Fig. 2, B) will show that any attempt to remove the sac in the ordinary way will result in mere removal of the sac from the main trunk of the funicular process, instead of from the abdominal peritoneum. Such an operation will be sure to fail because the upper opening of the funicular process will be left. The essential feature of the operation to be successful will ke the complete removal of the funicular process, together with the secondary sac, from the abdominal peritoneum. In order to do this efficiently it would be necessary to first separate the funicular process or its remains from the structures proper of the cord ; then to bring the hernial sac external to the epigastric vessels and remove it, together with the main portion of the funicular process.

Viscero-sacoular hernia. Hernice of the sigmoid flexure, coecum, and bladder. - These forms of hernia may be considered tozether, for the operative details that are essential to their treatment are identical. In both cases the organ involved has been drawn down with the peritoneum to assist in the formation of the funicular process. The close connexion that consequently exists between the bowel and the sac in such a case may be mistaken by an unpractised surgeon for adhesions. It is strange that although this form of hernia has been well studied by Lockwood and others I have noted a singular omission on the part of authors to describe the management of these cases; and this is not because the treatment is either self-evident or unimportant, as I have good reason to know. I have already dealt with this subject in a paper read before the Surgic al Section of the Australasian Medical Congress of 1902 at Hobart, Tasmania. The essential feature in the operation will be the cutting out of the portion of the sac with which the bowel is connected. In either case this will best be done by first clearly defining the whole of the sac and drawing it well out, then running the scissors up on either side of the bowel so far as the abdominal peritoneum; the bowel must now be returned into the abdomen together with the portion of the sac attached to it, and the remainder of the sac ligatured off and removed For this purpose the knot I have already described will be found very convenient. The sac in this case will in section prevent a hiatus at the part from which the bowel has been cut out. The hiatus, which is at the outer part of the sac will be conveniently closed by the first step in the insertion of the silk thread at A (Fig. 4).

Hernia of the bladder, while presenting some features of similarity to hernia of the sigmoid flexure and cxcum, differs in one essential particular - viz., that the bladder can be readily peeled off the sac, so that the surgeon bas the choice of two methods of dealing with it. I have operated upon four cases of viscero-saccular hernia of the sigmoid at the ages of $2,22,32$, and 68 years; upon one case only of viscero-saccular hernia of the entire cæcum in an infant, aged five months. All were satisfactorily managed in the way I have described above.

Femoral hernia.- The complete removal of the sac is, if possible, even more important in the case of femoral than of inguinal hernia. It is usually easy but it is necessary to carefully strip away every vestige of fascia from the neck, so that the sac can be freely pulled down. Usually the muscular wall of the bladder will be seen and should be pushed aside a little. When performed in this way the operation will be found to yield perfect results. That this fact has not been long since recognised is due simply to the fact that surgeons have not been impelled to deal with the sac in this thorough manner; hence have arisen a large number of different expedients for closing up the femoral canal. I notice with regret that they still continue to multiply; all are erroneous in principle and absolutely valueless in practice.

My object in this paper has been, not to write an exhaustive essay on hernia, but to display the changes wrought in the aspect in which hernia presents itself to the surgeon, and in the attitude of the surgeon towards hernia by the saccular 
theory. I am aware that I shall be asked for "results," and there is an incurable habit abroad in our profession of regarding resuits as in some way associated with "statistics." I accordingly give here the statistics prepared for my paper before the Surgical Section of the Australasian Medical Congress in November, $1905 .{ }^{6}$ An interval of one year from the date of operation was taken as the criterion of success. Britfly, out of a total of 89 cases of adult hernia I was only able to trace 63 . Of these 63 recurrence had taken place in three. These three recurrences require examining in order that their bearing on the saccular theory may be justly estimated. They are: 1. The case of direct funicular hernia to which allusion has been made in which I failed through ignorance of how to treat it. This is the only one of the three for which I do not bold myself to blame. 2. A man, aged 62 years, with strangulated left inguinal hernia which had just appeared for the first time. He returned five months later with a slight recurrence. (N.B.-It was a case of total funicular hernia and therefore can afford no evidence against the saccular theory.) 3. A case of ordinary inguinal hernia in a woman. Shortly after she left hospital she returned with a small recurrence. This is so absolutely contrary to all my experi. ence in this most simple class of cases that $I$ can come to no conclusion but that I must have blundered; in what way, exactly, I know not. So that of the three recurrences, we may fairly put the first two aside as not available in evidence against either the principle or the practice which I have been advocating.

I do not know whether these statistics compare favourably or unfavourably with those of other surgeons, because I do not know the statistics of other surgeons; nor am I particularly interested. The sole interest of my statistics lies in their bearing on the question of the truth of the saccular theory. I have always contended that the final and absolute means of testing the saccular origin of any individual case of hernia is to remove the sac. If the hernia have been an "acquired" hernia, recurrence will take place infallibly ; surely there can be no possible doubt as to that. If, on the other band, the hernia does not recur, then the sac must have been the cause of the hernia. I shall assume that these two self-evident propositions are accepter. This being so, the bearing of my statistics on the saccular theory is as follows. Out of 63 unselected cases of hernia in adults, 62 are proved to bave been of saccular origin and the remaining one provides negative evidence only. As a matter of fact, the odd one is really not worth discussing; there is no shadow of doulbt that it was identical in nature with the others I may add that I never consider it necessary to keep my patients recumbent for a prolonged period. They usually leave hospital within the fortnight with injunctions as to ordinary care and quietness for a week or two.

I need not say that removal of the sac for hernia is not a novel suggestion ; it is the oldest of the open operations for hernia because it is the most obvious. The history of the various operations for hernia since the introducion of antiseptic surgery is of extieme interest. It furnishes a striking commentary on the necessary supremacy of principles over practice; or better, on the chaos into which practice may fall if it should chance, as in the case of hernia, to fall under the dominance of a mistaken principle. Without attempting to review the various well known methods, I would like to point out that they seem to divide themselves into two groups : (1) an earlier grcup, of which the methods of Czerny, Bale, Macewen, and Kocher are types characterised mainly by differences in the management of the sac; and (2) a later group, Bastini, Halsted, and others-in these it is obvious that the main idea underlying them is that of increasing the strength of the inguinal region. Even the justly distinguished surgeon, McBurney, has a quaint mediæval notion of doing this by means of cicatricial tissue while at the extreme opposite pole stands a continental surgeon with the amazing suggestion of grafting a piece of dog's periosteum over the internal ring. It was owing to a grave $\mathrm{flaw}$ in the guiding principle that the first group failed to achieve complete success ; it is owing to precisely the same cause that the second group of "degenerates" ever saw the light. These, as they become elaborated, wander farther and farther from the true principles to which the first group were so very close.

The saccular theory bids us to abandon all these more recent devices; it further demonstrates to us the reason why

${ }^{6}$ Intercolonial Medical Journal of Australasia, Norember, 1905. a!l operations that have been hitherto devised have yielded imperfect results. It places the finger not merely on the defects of all operations for hernia: it denies that any formal operation such as could be associated with the name of a particular surgeon can be of real and comprehensive utility. It answers the obvious comment that will be made on this last statement to the effect that excellent results are obtained by various operations, by pointing out that the excellent results occur only in the cases in which the sac happens to be perfectly removed. It presents us incidentally with a curious seeming paradox; for while on the one hand it shows that there can be no such thing as an operation for hernia, it assures us, on the other, that there can be no such thing as a hernia that is not curable by operation. It promises practically perfect results in the future, far more perfect, at any rate, than have ever yet been attained by any operator or operation; but it imposes the three following conditions. Firstly, the surgeon shall concern himself with the peritoneo fascial layer of the abdominal wall alone. Secondly, be shall be pre. pared for the recognition of any and every variety of hernial sac, and ready with the appropriate measures for dealing with each. Finally (and this will follow naturally from the former two), he shall fully recognise that in the event of recurrence the explanation is not to be found in the weak. ness of the patient's muscles, but must be sought a little nearer home.

Melbourne.

\section{SO-CALLED TIRULENT SYPHILIS AND ITS TREATMENT.}

\section{BY FRANCIS J. LAMBKIN, L.R.C.P. \& S. IREL.,} COLONEL, R.A.M.C.

ALTHOUGH nowadays comparatively few cases of what used to be called malignant syphilis are met with compared with those which came under notice 20 years ago, still tbey are numerous enough to merit our keenest attention. As to the cause of this decline, it is supposed to be due chiffly to two factors: $(a)$ gradual attenuation of the syphilitic virus and $(b)$ the improved methods of treatment. By malignant syphilis is generally meant that form of the disease which is associated with an indurated sore, which, probably becomirg phagec ænic, is followed by rupial ulceration of the skir nodes, caries and ntcrosis of bone, and later by visceral lesions, cachexia, anæmia, and profound debility.

The histories of many epidemics show that the intensity of the virus of syphilis may be so great as to take on the above action irrespectively of the physical condition of the patient, attacking even those of the most robust constitution with the greatest virulence, and in spite of all treatment, but this latter is the exception. My on experience is that in the majority of cases there is present some well-marked cause which influences the progress of the disease. The following conditions, either singly or together, will generally be found to be associated in such cases: (1) poor physique ; (2) living under bad hygienic ald generally debilitating circumstances; (3) malaria; (4) the presence of any organic disease, especially renal ; (5) alcoholism ; and (6) delayed and infflicient treatment. As regards the first, it goes without saying that the poorer the constitution the less resistant it will be to the action of the syphilitic virus. Hygienic surroundings will certainly influence the progress of the disease for better or worse; thus the enervating climate of the tropics exercises a markediy baneful effect on it, while some of the worst cases are to be seen in overcrowded and dirty seaport towns. That malaria is a more potent factor in influencing syphilis for the worse than it has hitherto been given credit for is now certain, and, personally speaking, I can say that in some of the very worst cases which I have seen the patients had either been recently exposed to malaria at the time of syphilitic infection or the plasmodium could still be detected in their blood. The presence of any organic disease, especially renal, will, of course, exert a rowerful influence on the progress of any given case of syphilis, in being in itself not only a drain on the system but also by its presence either preventing altogether or at least limiting the exhibition of specific treatment. It has long been an accepted fact, and one that is true, that the drunkard has a worse chance of resisting the syphilitic virus than the moderate drinker. The last and probably the most important 